\title{
Development and Strategic Choice of Modern Agricultural Industry in Dazhou City based on SWOT Analysis
}

\author{
Yexing Sun ${ }^{\mathrm{a}}$, Qianqian $\mathrm{Ma}^{\mathrm{b}}$, Haosheng Li ${ }^{\mathrm{c}}$, Rongying Tang ${ }^{\mathrm{d}}$, Juan Wang ${ }^{\mathrm{e}}$, Huihan $\mathrm{Yu}^{\mathrm{f}}$, Peng Wu $\mathrm{Wu}^{\mathrm{g}}$, Zheming Zhang ${ }^{\mathrm{h}}$, and Siyi \\ Zhao $^{\text {i* }}$ \\ Dazhou Academy of Agricultural Sciences, Dazhou, Sichuan, China

\begin{abstract}
The development of modern agriculture in Dazhou City has both advantages and disadvantages, opportunities and challenges. By using SWOT analysis method, this paper analyzes the internal and external conditions faced by the development of modern agriculture in Dazhou City, and puts forward the rational strategic choice, which provides a reference for realizing the transformation from a big agricultural city to a strong agricultural city in Dazhou City.
\end{abstract}

\section{General situation of Agricultural Industry Development in Dazhou City}

According to the concept of "grasping the second production and promoting the third yield", Dazhou City has comprehensively promoted the scale, intensification, brand level of high quality grain and oil, selenium-rich tea, high quality fruit, characteristic culture, Chinese medicinal materials and other characteristic industries, and simultaneously promoted the development of leisure agriculture and rural tourism, and made great efforts to build a demonstration park for the integration of three industries [1].

According to data statistics, Dazhou City has built 30 state-level, provincial modern agricultural demonstration parks and $666.7 \mathrm{hm}^{2}$ demonstration areas, successfully established 2 provincial agricultural industry integration demonstration parks, 9 municipal modern agricultural industry integration demonstration parks, cultivated demonstration cooperatives at the provincial level of 62 , municipal level of 93, provincial family farms 18; We will build a standardized base of $146674 \mathrm{hm}^{2}$ of characteristic planting industries, cultivate 148 key leading enterprises, achieve sales revenue of 12.04 billion yuan, increase the processing rate of agricultural products to $50 \%$, and increase the commodity rate to $60 \%$, including 330 famous and excellent brands such as three products and one standard.

In 2018, the grain sown area was 555,100 hectares, the output was 3,171,300 tons, the first in the province; the oil crop planting area was 134,300 hectares, the output was 355,700 tons, the first in the province; the potato planting area was 162,700 hectares, and the output was 680,100 . Tons; vegetable planting area is 85,700 hectares, output is $2,829,500$ tons; fruit tree planting area is $819,900 \mathrm{mu}$, and output is 582,000 tons; ramie planting area is 16,700 hectares, output is 30,400 tons; Chinese medicinal planting area is 0.81 million hectares, yield is 2.14 tons 10,000 tons; the total output of meat is 456,800 tons, the output of aquatic products is 97,500 tons, the output of milk is 17,400 tons; the output of tea is 19,600 tons, and the output of sugar is 23,600 tons [2].

\section{SWOT analysis of modern agricultural development in Dazhou City}

SWOT analysis is a comprehensive and systematic analysis of the internal and external competitive environment and competitive conditions of the research object, so as to formulate the corresponding development strategy and countermeasures according to the research results [3]. S (strengths) is an advantage, $\mathrm{W}$ (weaknesses) is a disadvantage, $\mathrm{O}$ (opportunities) is an opportunity, $\mathrm{T}$ (threats) is a threat.

\subsection{Internal environment}

\subsubsection{Strengths}

Basic advantages. Dazhou is a big agricultural city in northeast Sichuan, rich in agricultural resources, and it is the hometown of Ramie, yellow flower, glutinous rice, Toon, Wumei, olive, selenium-rich tea and mash, which has laid a good industrial foundation for the development of agricultural products processing trade [4].

Location advantage. Dazhou City, located in the northeast of Sichuan Province and the combination of Sichuan, Chongqing, Hubei and Shaanxi provinces (cities), is an important transportation hub from east to north of Sichuan, a regional quiet sub-center, and also a trade and logistics center in Qinba area, with low transaction costs.

Market advantage. There are nearly 300 leading enterprises in the processing of agricultural products in Dazhou City, 6000 new agricultural management subjects 
have been cultivated, 330 brands of famous and excellent agricultural products, such as three products and one standard, have been established, and a perfect integrated production and management system of "production+ increase+marketing+trade" has been gradually built.

Policy advantages. The municipal government of Dazhou Municipal Committee attaches great importance to the development of modern agriculture, relies on the general grasp of rural revitalization, comprehensively promotes the development of characteristic agricultural products processing industry, and formulates and implements many policy measures, such as the development of "12335" and "3+6" characteristic industries, which provides a policy guarantee for the development of agricultural special products processing industry and the promotion of agricultural modernization.

\subsubsection{Weaknesses}

The constraints of land resources are obvious, and the agricultural infrastructure lags behind. There are more mountains and less land in Dazhou City, and the construction of agricultural infrastructure lags behind the needs of agricultural modernization, which restricts the development of agricultural scale, intensification and mechanization.

The majority of small and medium-sized enterprises in the processing of agricultural products, different industrial standards, non-standard technology, weak driving force of leading enterprises, less than $50 \%$ of the processing rate of agricultural products in the whole city, less than $50 \%$ of the contribution rate of agricultural scientific and technological progress, the lack of independent innovation ability of the processing enterprises and the lack of the core competitiveness of the market.

The industrial layout is scattered. The distribution of agricultural products processing industry in the city is scattered, and the distance between enterprises and raw material supply is longer, that is, it increases the transportation cost and transaction cost, and also brings many obstacles to the extension of industrial chain [5].

The ability to resist risks is weak. The risk of agricultural product production mainly comes from natural risk and market risk. Due to the change of climate conditions, the shelf life of agricultural products is short, the storage and inventory loss rate of enterprises is increased, and the development of agricultural products logistics is not enough to meet the total needs of the market. At the same time, the imperfect market mechanism also reduces the income of processing enterprises.

\subsection{Exotic environment}

\subsubsection{Opportunities}

The state attaches importance to the issue of agriculture, rural areas and farmers. On the one hand, since 2004, the first document of the Central Committee has taken the issue of "agriculture, countryside and farmers" as the focus of national attention for 15 years in a row; on the other hand, the agricultural supply-side structural reform and the implementation of the rural revitalization station strategy have further promoted the transformation of agricultural products processing from quantitative growth to quality improvement, factor-driven to innovation-driven, and decentralized layout to cluster development.

The promotion of industrial development strategy. The formulation of the development strategy of the agricultural product processing industry, the promotion of the innovation-driven strategy, the implementation of the brand strategy of "three products and one standard", and the formulation of the strategic plan of rural revitalization in Dazhou City will have a profound impact on the processing of agricultural products, the improvement of product quality, the development of agricultural products market and agricultural economic benefits.

\subsubsection{Threats}

The pressure of competition in the external market has increased significantly. Due to the new normal of economy, the aggravation of external open competition and the potential influence of trade friction between European and American countries, coupled with the fierce competition of competitors in the region and the impact of foreign commodity prices, the development of agricultural products processing industry in our city is facing great threats and challenges.

The pressure on the development of modern agriculture has increased. Agricultural production waste is difficult to deal with, chemical pesticide residues, ecological environment damage and other problems, resulting in increased pressure on environmental protection, increased agricultural production costs, agricultural processing enterprises brand identification and product quality standards are more difficult, especially the imbalance of rural human structure, the age of producers, the lack of professional farmers, the development of modern agriculture is difficult [6].

\section{Strategic choice}

\subsection{Development positioning}

Our city should make full use of external opportunities to improve the development level of its own agricultural products processing, combine with the internal and external development environment, accurately locate the development of regional characteristic agricultural products processing industry from the actual situation, rely on the rich agricultural products resources in Dazhou City, focus on the development of grain and oil processing, characteristic livestock and poultry products processing, selenium-rich tea processing, characteristic fruit and vegetable processing, Ramie processing, Chinese medicinal materials processing, We should innovate and cultivate the development model of characteristic industries dominated by "Internet intensive processing", support the establishment of regional brands of "selenium-rich" and "characteristic" agricultural products, highlight the non-replicability of regional industrial characteristics, actively adopt new Internet technologies, 
change the management mode of agricultural products processing industry, and comprehensively promote the transformation of agricultural products processing industry from resource advantage to industrial advantage and from comparative advantage to competitive advantage [7]. Combine traditional agriculture with dominant and characteristic agriculture organically, persist in winning by characteristics, and on the basis of persisting in development practice, strive to deepen the strategic adjustment of agricultural products processing industry structure, optimize the allocation of agricultural industry resources, and promote the cluster, scale, brand and collectivization development of characteristic agricultural products processing industry in Dazhou City [8].

\subsection{Strategic choice}

According to the above SWOT analysis of modern agricultural development in Dazhou City, we can briefly summarize four strategic combinations, namely: SO growth strategy, ST transfer strategy, WO development strategy, WT defensive strategy [7]. The four combination strategies should be tailored to local conditions and implemented by classification.

The growth strategy of $\mathrm{SO}$ is suitable for the characteristic agricultural industry and competitive advantage with absolute advantage in the region, and the processing enterprises are in the rising period. On the one hand, according to the market demand and the reality of industrial development, the government can determine the overall layout and construction scale of characteristic industry development, plan the economic belt of industrial development, implement regional specialized production, cooperate with the industrial gradient development strategy, and establish the industrial cluster processing park [9]. On the other hand, the leading processing enterprises, the production enterprises with obvious competitive advantage, should seize the opportunity, adopt the differentiation, characteristic, brand product development strategy, and strengthen the enterprise strength with technological innovation.

ST transfer strategy is applicable to the characteristic agricultural industry with relative advantages and processing enterprises with relatively high competitive advantages. On the one hand, the government should guide the enterprise to re-plan the development pattern, promote the ecological development of industry, scientization and standardization, rely on the modern enterprise management and management management system to ensure the product quality, highlight the quality of the products "selenium-rich" and "organismundefineds habits", transform the comparative advantage into a competitive advantage, and realize the transformation of agricultural products from quantity growth to quality improvement; On the other hand, processing enterprises with relatively high competitive advantages should adopt diversified product strategies, speed up R\&D of high-quality new products, extend the industrial chain, promote enterprise efficiency increase, do a good job in enterprise brand building, and launch after-sales service "a gilded signboard".
The WO development strategy is adapted to the unique advantages, but because of the lack of the regional advantages, the development of the slow-growing one-page industry and the transformation and upgrading of the processing enterprises. In this regard, the government should be inclined to policy, to strengthen the construction of agricultural infrastructure, to set up regional advantageous industrial base, to encourage the implementation of standardized scale production, to further optimize the marketing channel, to strengthen the degree of risk supervision and macro-control, and to promote the development of the industrial advantage [10]; The processing enterprises should speed up the transformation and upgrade, optimize the product structure, increase the investment and investment, deepen the scientific and technological strength, and strengthen the development of the enterprise.

WT defensive strategy is suitable for processing enterprises with low competitiveness and high homogeneity of products. In this regard, the government should replan the development of regional agricultural industry, take classified measures according to local conditions, and focus on building industries with obvious advantages in the region. Enterprises should consider their own development in the long run, carefully select the target market, promote mergers and acquisitions by improving scientific and technological innovation, attracting investment, cultivating regional brands and leading enterprises, and promoting mergers and acquisitions [11].

\section{References}

1. H. M. Sun, J. Zhang, C. R. Yu. Convergence of the three industries and agricultural efficiency advancement: Empirical analysis based on WIOD data [J]. Journal of Chinese Agricultural Mechanization, 2018, 39(11): 100-107.

2. 《Dazhou Yearbook》Compilation committee. Dazhou Yearbook [M]. Sichuan Science and Technology Press, 2018, 12.

3. Z. H. Kang, H. X. Chen, H. Z. Liu. Choice of modern agricultural development strategy of Xinghua city in Jiangsu province based on SWOT analysis [J]. Management of Agriculture Science and Technology, 2012, 31(01): 15-17.

4. Y. J. Wang. Talking about the present situation and development countermeasures of modern agriculture in Dazhou city [J]. Cultural Geography, 2015(14): 279.

5. T. Yang. Analysis of agricultural products value chain in agricultural industry chain integration [J]. Commercial Times, 2018(12): 150-152.

6. L. C. Du. The current situation and development countermeasures of rural food processing industry clustering in china in the new period [J]. Agricultural Economy, 2017(5): 57-58.

7. L.J. Wang, Y. Liu, J.Q. Fei, J. Yang, B. Hu. Development paths of sustainable agriculture in the key ecological function oriented areas based on SWOT analysis-taking zhejiang province as an 
example [J]. Chinese Journal of Agricultural Resources and Regional Planning, 2016, 37(11): 65-71.

8. Z. L. Li. Strategic choice of modern agricultural development in Jingtai county based on SWOT analysis [J]. Gansu Nongye, 2013(20): 3-5.

9. Z.Y. Jiang. SWOT analysis and countermeasure research on Xinjiang tourism agriculture development [J]. Agricultural Economy, 2016(04): 33-35.

10. T. Ren. Research on the basic path of china's rural economic development from the perspective of modern agricultural industrial system construction [J]. Agricultural Economy, 2019(07): 27-29.

11. X.M. Guo, Y.B. Dai. Macro thinking of development of modern agriculture in Sichuan and crucial breakthrough [J]. Rural Economy, 2008(05): 61-64. 